\title{
LA CIENCIA LINGUISTICA, UNA DOBLE EXPECTATIVA PARA EL DOCENTE DE LENGUAS
}

\section{Lucia Tobón de Castro**}

La falta de presencia de la universidad en la solución de los problemas nacionales o el divorcio que existe entre los planes de desarrollo propues tos a nivel nacional y las metas y propósitos que orientan el trabajo universitario son temas que a todos preocupa. Y como corolario de esta inquietud general, es frecuente escuchar a quienes se dedican a la actividad intelectual formularse preguntas como: ¿por qué la universidad colombiana no ha hecho de la investigación un propósito institucional? Ó ¿por qué la investigación no es parte fundamental del diario quehacer de las instituciones universitarias en Colombia?

Son múltiples las respuestas que se han intentado, todas ellas inspiradas por marcos coyunturales, por visiones segmentadas del problema o por enfoques particulares de lo que debe de ser la gestión de la educación avanzada en el país. De ahí que a menudo se traigan a cuento razones como la carencia de recursos, sean ellos materiales o humanos; la falta de interés de los colombianos por la producción de conocimientos; la densidad de los curricula que se ofrecen; la deficiencia en los procesos de admisión y qué se yo de otras cuantas justificaciones que se esgrimen. Mas parece que, manera quizás inconsciente, todos tratásemos de soslayar el problema embrollándolo y desviando atención hacia aspectos secundarios o transitorios sin llegar a tocar el fondo de la situación.

Esta visión distorsionada de la realidad, ha dado lugar a distintas actitudes de parte de las universidades particulamente las públicas- que, a mi modo de ver, deberían encarar el asunto como una preocupación nacional por cuanto son instituciones que desarrollan políticas oficiales. Veamos algunas:

1. Se observa la tendencia a registrar como planes institucionales proyectos y subproyectos individuales o de grupos, inspirados en la vocación personal de los investigadores o en sus particulares intereses académicos y/o científicos, pero que de ninguna manera representan un plan articulado de investigación.

2. De otro lado, hay quienes esperan que la producción científica sea siempre trascendental y opere sobre as pectos o hechos cruciales que determinen vuelcos en el proceso de conocimiento, olvidando que el saber se construye paso a paso con el trabajo cotidiano tranquilo y pausado apuntando fimemente hacia una

\footnotetext{
*Este artículo fue leído delante de los profesores y estudiantes del Departamento de Lenguas de la UPN. Abril 17, 1990

${ }^{* * *}$ Profesora de Lingüística, Universidad Pedagógica Nacional.

${ }^{* * *}$ Yunis, Emilio. ¿Están los colombianos bien informados? Bogotá: El Espectador. Junio 4 de 1990.
} 
meta. Los despliegues publicitarios no son propios de los hallazgos científicos, puesto que los resultados de este quehacer suelen presentarse en foros que los valoren y divulguen, poniéndolos al alcance de todos los públicos.

3. Surge de aquí una tercera actitud simplista que cree ver ciencia donde no la hay, por cuanto concede al desarrollo de tecnologías el valor de creación científica y es así como apropiándose teorías logradas en otros país es reducen su trabajo a procesos de refomulación de principios o al desarrollo de técnicas de aplicación empírica. ¡Vaya investigación!

Hechas estas consideraciones sobre la situación actual de la investigación en las instituciones de nivel superior, es oportuno dar respuesta a las preguntas planteadas al principio de mi exposición, respuestas que nunca podremos hallar al interior de nuestras universidades por cuanto se trata de un fenómeno que tras ciende al ámbito universitario y se ubica en el marco conceptual que ilus tra el sistema educativo en todos sus niveles. Consiste en que la creación de conocimiento científico no es propósito nacional, el país carece de vocación investigativa como soporte de la gestión educativa. Tradicionalmente se ha planeado el desarrollo a partir de la transferencia de tecnologías que resulta más expedita y no requiere las grandes inversiones que la investigación exige. Para probar la veracidad de este acerto basta señalar algunos hechos: 1. La Universidad sigue conservando su esquema de escuela profesional; 2 . La investigación es tratada como una actividad suntuaria a la cual se le asignan partidas mínimas en los presupuestos universitarios, diríamos que ella se nutre de lo que las directivas de turno "logren pellizcar" de otros rubros del funcionamiento general; 3 . No se desarrolla la vocación investigadora de la juventud des de los primeros niveles de la educación, por cuanto se desconoce a los docentes el papel importante que tienen en este proceso y no se les forma para que hagan de la investigación el fundamento de su acción educativa.

Las anteriores consideraciones me han llevado a reflexionar sobre la importancia que tiene la investigación para quienes hemos hecho de las ciencias del hombre meca de nuestro trabajo intelectual y de la ciencia del lenguaje foco de nuestro quehacer universitario. Por esto he decidido llamar la atención de quienes se están formando para ejercer la profesión de docentes en lenguas en cualquiera de los niveles del sistema educativo colombiano sobre nuevas expectativas que el estudio de la lengua y de las lenguas ofrece. De manera particular a dos de ellas que, superpuestas a la condición de educadores, enriquecen la fundamentación teórica del futuro maes tro.

Tomaré como punto de partida de este escrito un concepto que aparece esbozado, quizás de manera soslayada, en el ensayo que publiqué en la revista Forma y Función. Número 4, de la Universidad Nacional, que dice: la ciencia es un proceso de reflexión sistemática mediante el cual el hombre busca apropiarse de su entorno, sea él natural o social, a fin de lograr la satisfacción de sus necesidades, y no la simple respuesta a la curiosidad como se creyó en otro tiempo. A este respecto se han presentado diversos criterios sobre la llamada 
teoría de la necesidad. Hay quienes consideran que son básicamente dos: la necesidad de conocer para satisfacer su curiosidad y la necesidad sentir para asegurar el disfrute tanto físico como espiritual. Otros, entre ellos Maslow en su leona de La motivación humana ${ }^{1}$ reconocen cinco clases de necesidades: biológicas (basadas en el instinto de supervivencia); sociales (manifiestas en los procesos de integración al grupo); de seguridad (referidas al dominio del medio); de estima (expresadas en procesos de conducción y manipulación) y de autorrealización (dirigidas a identificar desarrollar y poner en juego sus personales capacidades y habilidades ). (Ver Fig 1)

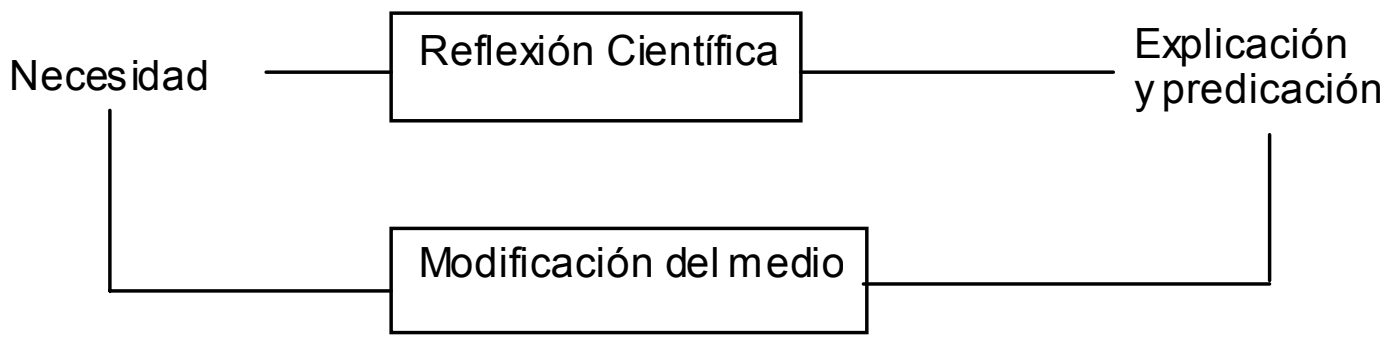

Figura 1

Ahora bien, sean cuales fueren los diversos tipos de necesidad que el hombre experimente, para satisfacerlos necesita: bien transformar el medio bien cambiar el entomo o bien adecuarse a él mismo si es su propio yo el que los suscita. Y el único camino es conocerlos y conocerse a fin de poder explicar los fenómenos que unos y otro en cierran, es decir, el mundo y su mundo. Como bien dice Spirkin la última etapa en el proceso de desarrollo cognos citivo del hombre (tanto a nivel filogenético como ontogenético) es aquella en la cual se vuelve sobre sí mismo convirtiéndose simultáneamente en sujeto cognoscente y en objeto conocido ${ }^{2}$. Resultado de esta búsqueda de leyes principios que regulen los fenómenos es la ciencia, conjunto de teorías que debe trascender los hechos y lograr tal grado de abstracción y generalización que llegue a explicar y predecir el comportamiento de esos hechos. De ahí que todas las disciplinas científicas -ocúpense de la naturaleza o de la sociedad-, sean procesos reflexivos del hombre en los que se integran sistemáticamente diversos elementos que, partiendo de los postulados ya establecidos, registran los hechos, los conceptualizan, fomulan hipótesis que deben ser contrastadas y verificadas, y construyen estructuras teóricas formalizadas que pueden tener el carácter de principios o de leyes (Ver Fig. 2).

\footnotetext{
${ }^{1}$ Maslow, A. H. Teoría de la motivación humana, Chicago: The University of Chicago Press, 1967.

${ }^{2}$ Spirkin A. G. Origen del lenguaje y su papel en la formación del pensamiento. México: Editorial Grijalbo SA. 1966.
} 


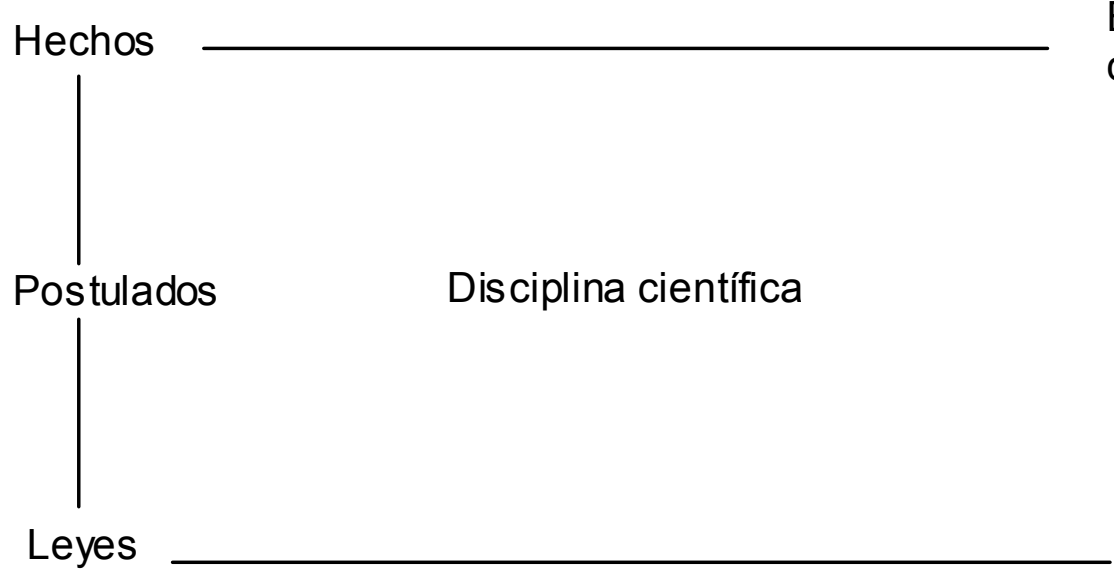

Estructuras conceptuales

Hipótesis<smiles>C1CCCCC1</smiles>

Estructuras teóricas

Figura 2

La organización de este proceso y los sistemas de formalización empleados han dado lugar a los diversos modelos científicos utilizados por la ciencia para lograr la objetividad, asegurar la validez de los resultados y garantizar el nivel de generalización alcanzado por sus formulaciones teóricas. Un desarrollo que se inició con modelos de descripción cualitativa o cuantitativa de los hechos, ya hoy ha logrado rebasar la inferencia inductiva clasificatoria o taxonómica y dar paso a la inferencia deductiva cuya meta es la explicación y la predicción.

Después de este somero esbozo sobre el objeto y la metodología de la ciencia, meditemos un poco sobre la que constituye el soporte teórico de nuestra gestión de docentes de lenguas: la lingüística. Comencemos por situarla en el ámbito de la ciencia y por señalar el objeto de que se ocupa esta dis ciplina, indiscutiblemente el milagro del siglo XX en lo que se refiere a los estudios del hombre.

Como es bien sabido, la lingüística es la ciencia del lenguaje que, después de haber comenzado hacia 1910, su proceso investigativo empleando el método de la sociología, actualmente cuenta con múltiples modelos científicos desarrollados por los investigadores de acuerdo con el marco filosófico en que se sitúan y con el objetivo que persiguen. No me detendré a analizar los diversos modelos investigativos propuestos por la lingüística porque ya me ocupé de ellos en otra oportunidad y a todos y cada uno reconozco méritos por cuanto han sido instrumentos de trabajo científico y gracias a los resultados que ellos produjeron y están produciendo ha sido posible la conformación de una disciplina sistemática y objetiva que cuenta con un cuerpo de teorías que han logrado un nivel explicativo y predictivo acerca de una de las facultades humanas, el lenguaje. Como bien lo señala Rona, "un modelo es una estructura conceptual que trasciende la realidad, es el esquema teórico que surge del método seleccionado por el investigador y 
sólo tiene vigencia como instrumento de investigación" "3. Fuera de este ámbito no tiene aplicación, ni puede ser transferido al ambiente pedagógico y utilizado como diseño ins truccional.

Pero, ¿en qué consiste la facultad del lenguaje? y ¿qué papel desempeña la lingüística en lo que a ella se refiere? La facultad del lenguaje es esa condición particular propia de esa especie es pecífica ${ }^{4}$ que llamamos homo sapiens, cuyas condiciones biológicas le permiten crear un mundo conceptual -remedo del mundo real en que se halla inmerso- e inclusive generar estructuras conceptuales de su propia invención que son substrato de su experiencia vivencial y no tienen referente en el mundo de los objetos o de los hechos. Es más, gracias a ella el hombre jerarquiza y almacena conceptos que puede evocar, enriquecer o rectificar a su amaño. Y como si esto fuera poco es elemento esencial en la construcción del significado, medio sin igual para transfomar y sublimar el mundo de la realidad. Pero no termina aquí la proyección de la facultad humana del lenguaje, es ella la que permite al hombre hacer asociaciones y establecer relaciones entre diversas estructuras conceptuales e idear sistemas simbólicos para representarlas o comunicarlas si así lo desea. Pues como bien dice Benveniste ${ }^{5}$ no es función exclusiva de los signos comunicar, es posible que el hombre los produzca para sí o simplemente para evocar la realidad.

También es papel del lenguaje la organización de nuestros procesos de pensamiento, que pueden manifestarse a través de la lengua o mediante otro sistema simbólico: una ecuación matemática, un diseño, un gráfico, por ejemplo. El hecho de que de Saussure haya reducido el lenguaje a la lengua para, en su momento, as egurar el principio de unidad y poder ubicar a la lingüística dentro de las ciencias que estudian los hechos humanos ${ }^{6}$, no quiere decir que sigamos en esta confusión entre lo que es facultad, potencialidad humana el lenguaje- y lo que es su concreción, su realización -la lengua-.

Pues bien, corresponde a la lingüística descubrir y formalizar las leyes que explican el fenómeno lenguaje en el hombre y que, por tanto, constituyen metaleyes para todos aquellos sistemas sígnicos (verbales y no-verbales) que él mismo ha ideado y en los cuales se halla inmerso. El hecho de que en sus procesos de contrastación y verificación la lingüística tenga que acudir a los sistemas semiológicos, específicamente a las lenguas, no quiere decir que su campo de investigación esté ahí y termine ahí. Simplemente desciende a la realidad de los signos para verificar la validez de sus hipótesis, pero regresa al mundo oculto del lenguaje que es el objeto de sus preocupaciones. No olvidemos que los hechos de realidad son solo evidencias de las leyes que a ellos subyacen.

\footnotetext{
${ }^{3}$ Rona, J.P. La concepción estructural de la sociolingüística, La Haya: Mouton, 1970.

${ }^{4}$ Lenneberg, L. Una perspectiva biológica del lenguaje, Madrid: Revista de Occidente. Ediciones Castilla S.A.,

${ }^{5}$ Benveniste E, Problemas de Lingüística General. México: Veintiuno editores, S.A. 1974.

${ }^{6}$ Benveniste E. Ibidem.
} 
Son innumerables los problemas que esperan una solución de parte de los lingüistas investigadores, todos ellos de gran importancia por cuanto el lenguaje está presente en todas las vivencias humanas; es factor indispensable en los procesos cognoscitivos del hombre; es responsable del desarrollo de su capacidad abstractiva y elemento básico de auto-gestión por cuanto al facilitarle la formación de un esquema mental del mundo le permite adquirir la dimensión de su entorno y su propia dimensión. Está, pues, abierto el campo para quienes es tén interesados en encarar alguno de estos problemas y adelantar un proceso investigatiyo, aporte que buena falta le hace a Colombia.

He aquí la primera expectativa que se ofrece al docente de lenguas, pues, contrario a lo que piensan las autoridades educativas de nuestro país, sólo puede guiar a otros en el proceso de adquisición del conocimiento (gestión del docente) quien, habiendo participado en sus procesos de creación puede idear es trategias para ayudar a otros a apropiárselo e interpretarlo, lo cual constituye en realidad una doble dimensión de investigación científica.

La lingüística, como ciencia factual, tiene un referente objetivo inmediato que aparece implícito en el contexto de la teoría y otro mediato explícito en el proceso de contras tación con la realidad. Todo modelo lingüístico por abs tracto que sea es una idealización, o si se quiere un boceto conceptual, de algún objeto cuya existencia supone. Aunque una teoría lingüística seleccione una clase particular de sistemas lingüísticos ( $x$ lengua) y la represente en forma simbólica $y$ simplificada más bien que icónica y completa, lo que está representando es una realidad factual existente. La referencia objetiva mediata es, por tanto, la correspondencia entre un modelo conceptual y un objeto real, es decir la representación que el modelo hace de la realidad (Ver fig. 3$)^{7}$.

7 Esquema inspirado en el que propone Bunge en su ensayo Teoría y realidad. Barcelona: Editorial, S.A., 1985. 
Teoría

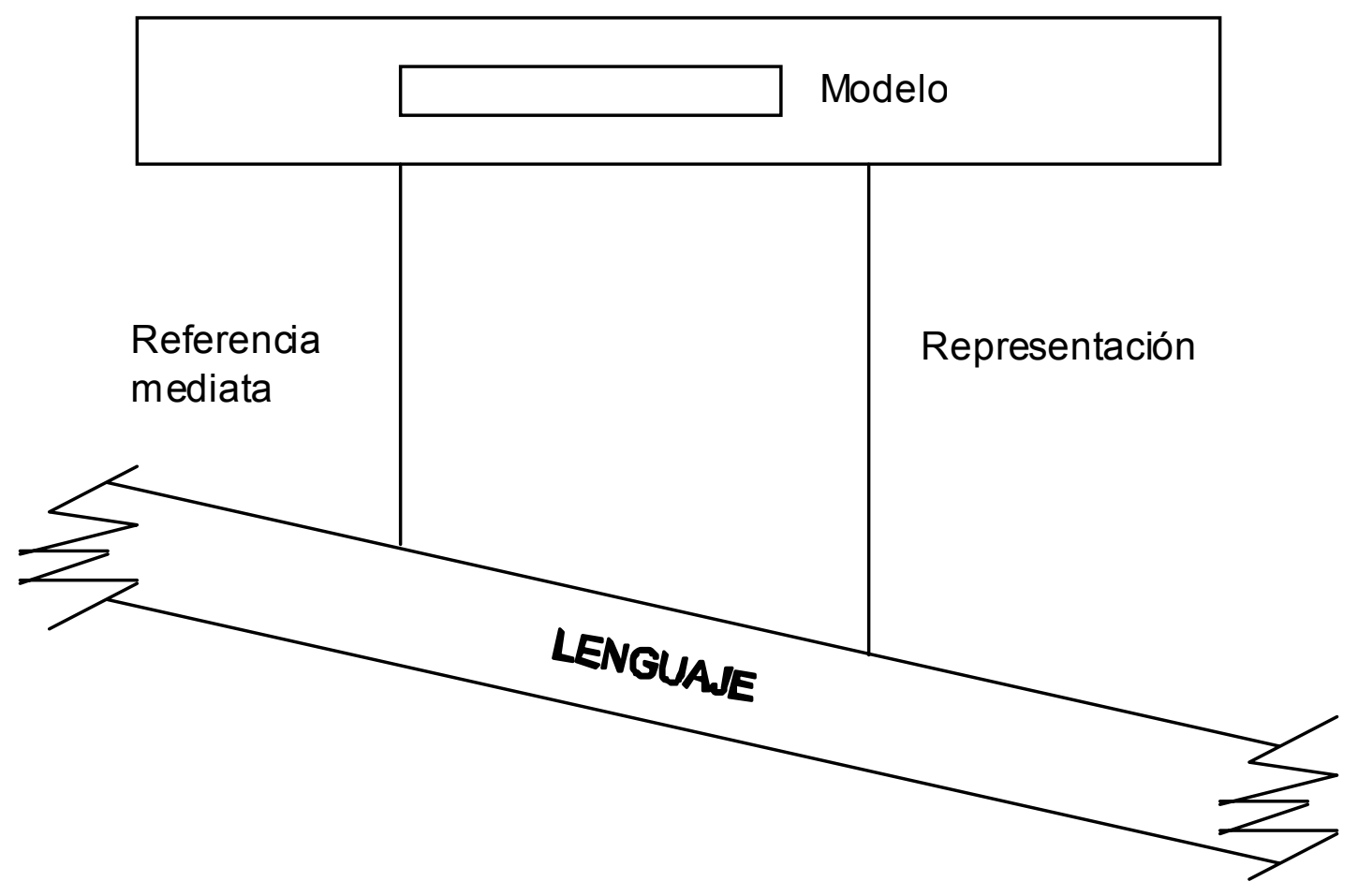

Sistemas Lingüísticos

Figura 3

Esa referencia objetiva mediata puede ser observable o inobservable y partiendo del modelo se llega a ella mediante procesos racionales deductivos, o bien, es un hecho observado del cual puede partir el investigador para generar su estructura hipotética (inferencia inductiva) la cual es verificada en la realidad (inferencia deductiva), que sirve de medio de contras tación, es decir, actúa como evidencia ${ }^{8}$.

${ }^{8}$ Bunge Mario, op. cit., 1985. 
Teoría

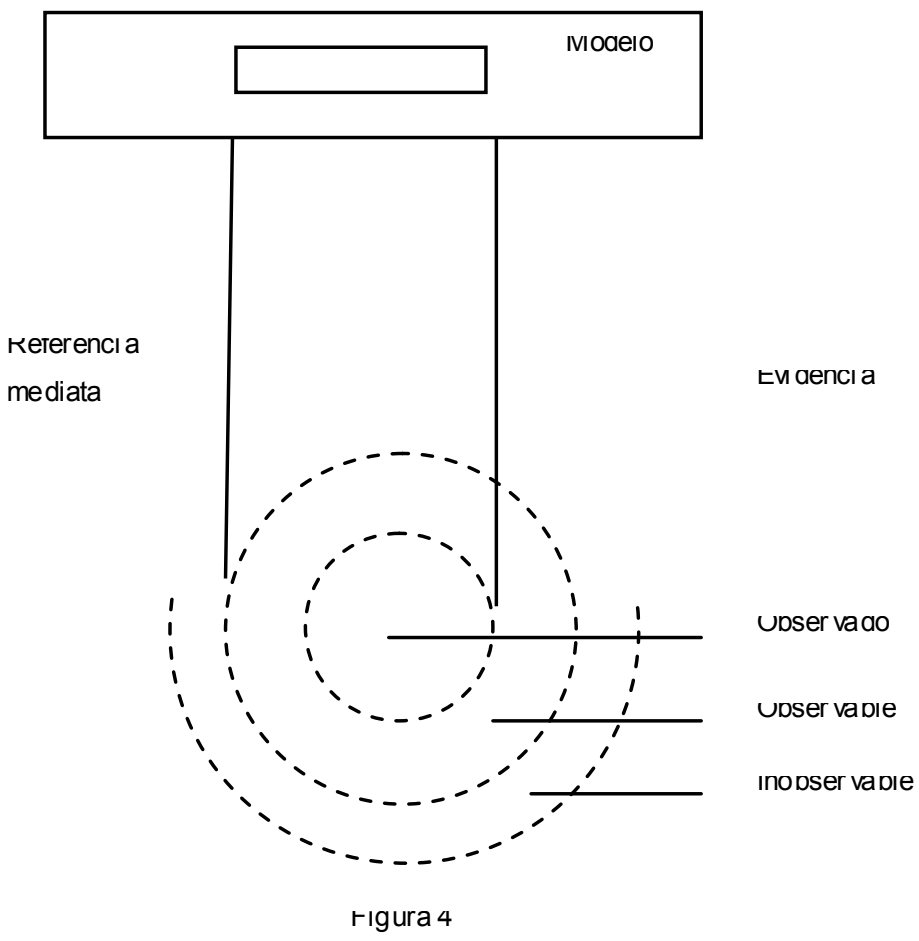

Un objeto, un hecho, una situación, es decir, una realidad observada sólo adquieren el valor de evidencia cuando entra a ser medio de confrontación de un sistema de hipótesis que ha sido construido a partir de un objeto inobservable (vgr., los procesos conceptuales que dan origen a la producción lingüística) u observables (por ejemplo, los contenidos afectivos que se reflejan en la expresión lingüística).

Lo anterior nos muestra una nueva fase de la lingüística: la lingüística aplicada que se ocupa del estudio de las lenguas en particular y cuya función es la construcción de las gramáticas, modelos empíricos que, mediante la aplicación de los principios formulados por la ciencia teórica, explican las relaciones que se dan al interior de la estructura sistémica de las lenguas, es decir, la relación signoreferente, la relación signo-signo y la relación signo-usuario, las cuales son estudiadas por las tres partes de la gramática: la semántica, la sintaxis y la pragmática. Esta concepción, que ha superado el nivel de la lingüística oracional y ha agregado al estudio de la lengua como saber, el anális is de la lengua como instrumento de comunicación, identifica tres unidades estructurales del sistema: la proposición como unidad lógico-semántica, la oración como unidad formal abstracta $y$ el enunciado como unidad pragmática. Se reconoce además que en los procesos de producción e interpretación de unidades discursivas, el hablante aplica no solo la noma lingüística del sis tema como propone Coseriu sino también una noma psicosociopragmática puesto que la lengua como todo sistema semiológico cumple, en el proceso de interacción humana, una función que 
permite a un individuo desarrollarse como persona y as umir una personalidad por cuanto sabe como actuar en una determinada situación y qué formas lingüísticas tiene que utilizar en el entorno en que se encuentra.

Presento a continuación dos esquemas que permiten visualizar la forma como se ha concebido la lengua en función. En primer lugar el punto de vista de Coseriu para quien cada vez que un hablante (usuario) da forma concreta a una lengua (habla) aplica una noma lingüística resultante de la estructura interna del sistema y del saber de la comunidad.

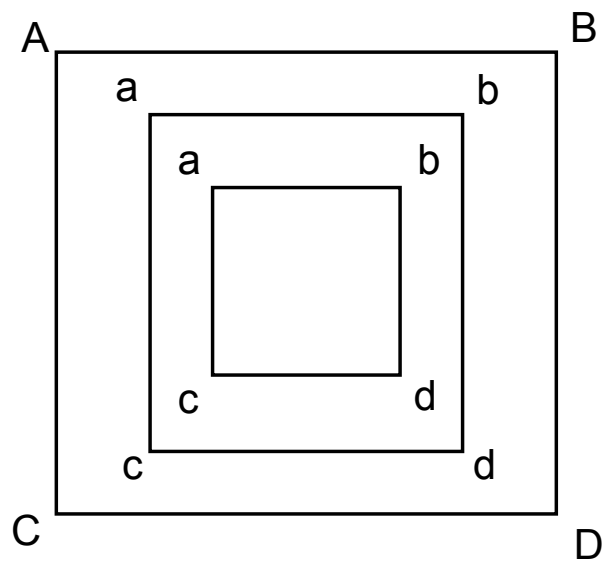

A, B, C: habla

A, b, c: Norma

A, b, c: Lengua

Figura 5

Y en segundo lugar una propuesta en la cual recojo el pensamiento de Coseriu pero reconozco la existencia de una noma externa al sistema que bien podría ser norma psicosociopragmática conforme la intuye Dittmar en su modelo.

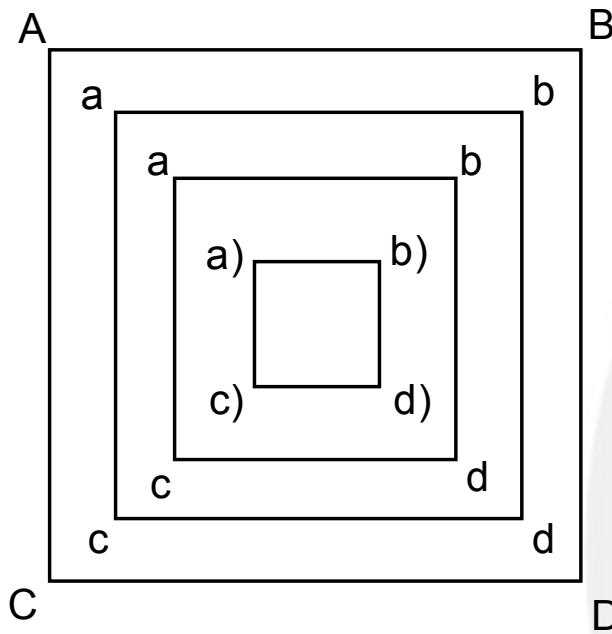

A, B, C, D: Norma psicolingüística A, b, c, d: habla

$A, b, c, d:$ Norma lingüística

$A, b, c, d$ : Lengua 
Derivadas de esta concepción han surgido dos propuestas de análisis del proceso comunicativo: la lingüística textual y el análisis del discurso, cada una de las cuales es la aplicación de los principios generados por la investigación realizada en el marco de la lingüística teórica.

En el ámbito de cada código verbal también encuentra el docente de lenguas amplio campo para la investigación tanto de la estructura semántico-pragmática del sistema como de las variaciones socio y psico-pragmáticas a que éste está sometido, debido a presiones emocionales, sociales o culturales tales como reacciones subjetivas, prejuicios, creencias, normas de comportamiento social, tradiciones etc., campo en el cual han surgido nuevas disciplinas, relativamente autónomas, como la psicolingüística y la sociolingüística, las que según Hymes deben desaparecer cuando la lingüística llene a cabalidad su doble función de ciencia teórica y empírica, que analice el acto lingüístico como realización psicosocio-pragmática.

No menos importante sería su aporte en la propuesta de diseños instruccionales inspirados en el marco teórico y epistemológico de la ciencia del lenguaje, diseños que a la vez que fuesen respuesta a las necesidades educativas de nuestras gentes teminaran con la transferencia tecnológica de esquemas creados para otras culturas y otros contextos.

El esbozo anterior nos conduce al planteamiento que representa la tesis de $\mathrm{mi}$ exposición: La ciencia lingüística constituye una doble expectativa para el docente de lenguas por cuanto es objeto de su reflexión nomológica y soporte teórico de su realización nomopragmática.

Existe por tanto un amplio es pectro de participación del docente de lenguas en el campo de la investigación científica (tanto teórica como pragmática) y pedagógica que le permitirá su perfeccionamiento y enriquecimiento permanentes, pues to que el desarrollo actual requiere educadores cultos, conocedores profundos del saber que enseñan. No de otra manera se logrará elevar el nivel de la cultura lingüís tica de nuestra juventud, sumamente pobre en la actualidad.

Se preguntarán ustedes por qué en nuestro país no se han aprovechado todas estas posibilidades que ofrece la lingüística. Dejando de lado el problema que esbocé en la introducción, me referiré a problemas concretos. Hay dos razones para que el gran desarrollo de la lingüística no haya causado en el estudio de las lenguas (sea la propia o las segundas lenguas) un progreso tan relevante como el que la matemática moderna generó en el estudio de es ta disciplina.

En primer lugar, el gran cambio que significó el descubrimiento de que los problemas humanos eran susceptibles de ser rigurosamente analizados por disciplinas científicas que daban al traste con la postura especulativa de las humanidades. Yo diría que aún queda en Colombia, mucha gente medianamente culta que sigue viendo la realidad humana como ensueño y no como problema que hay que explicar y resolver. Todavía se siguen mirando las ciencias del 
hombre o las ciencias sociales como disciplinas de segundo orden frente a las ciencias de la naturaleza.

$Y$ ya en un campo más específico, el desarrollo de la ciencia lingüística ha causado una gran confusión entre los docentes y es así como los modelos lingüís ticos creados por los investigadores para hallar explicación a los fenómenos del lenguaje bien como rasgo de la humanidad bien como una realización concreta, medio de comunicación de una comunidad, han sido tomados como diseños o modelos pedagógicos y llevados al salón de clase, cuando lo que conforma una ciencia es el conjunto de teorías ya comprobadas y validadas, que constituyen el conocimiento que ella ha creado y que debe ser transmitido de generación en generación. Amén de esta confusión, se ha incurrido en actitudes subjetivas que han llevado al docente a privilegiar unos modelos sobre otros dejándose guiar por las influencias foráneas y olvidando que su gestión debe estar dirigida a proponer diseños instruccionales que a la vez que hagan comprender a sus alumnos los principios de la ciencia garanticen el perfeccionamiento y enriquecimiento de sus procesos de producción e interpretación lingüística. Recordemos que el maestro como investigador debe crear modelos de investigación teórica o empírica y como docente debe idear diseños instruccionales que integren la teoría a los procesos de aprendizaje.

Por último no quiero pasar por alto la enome contradicción de las autoridades educativas del país que niegan a las facultades de educación, la opción de realizar proyectos investigativos en ciencias de la naturaleza y en ciencias del hombre. En otras palabras se les propone que hagan investigación educativa, sin un soporte científico teórico. Es indudable que esto redundará en el hecho de que la enseñanza de las lenguas y la reflexión sobre la lengua propia terminen por ser una actividad puramente artesanal que no des pierte el interés de la juventud.

Espero que mi propuesta sirva de tema de reflexión para las nuevas generaciones de docentes de lenguas que han de ser las encargadas de reorientar la educación en Colombia. 